\title{
WAR INJURIES OF THE SPINE AND SPINAL CORD.
}

\author{
By GEOFFREY KNIGHT, M.B., B.S., F.R.C.S.
}

(Surgeon, Emergency Medical Service; Surgeon, West End Hospital for Nervous Diseases; Neurological Surgeon, Metropolitan Hospital, London, Princess Elizabeth Hospital for Children, and Southend General Hospital; Assistant for Neuro-Surgery, British Post-Graduate Medical School.)

A high proportion of the spinal injuries occurring in war time will be of the closed variety, comprising fractures and fracture dislocations of the vertebral column resulting from accidental injury. For example, from falls down the hatchway, into craters, in civilian injuries from the crush of falling masonry, or in battle from the blast of high explosive hurling patients into grotesque postures, or causing the collapse of parapets and sandbags with resulting crush and flexion injuries.

Open wounds of the spine, compound fractures with or without division of the spinal cord will be met with relatively seldom, for these injuries often prove rapidly fatal, not only on account of shock and hæmorrhage, but more especially from the fact that few such wounds can exist without grave associated injuries of the abdomen or chest, unless a bullet by evil chance should strike the spine exactly. In addition, there will be cases in which the symptoms of an injury of the spinal cord co-exist with adjacent lesions which have not directly involved either the cord or the vertebral column, a group which may at times produce considerable confusion. In all of these, as with injuries of the head, the associated injury of the nervous system dominates the clinical picture and is all-important for the future well-being of the patient. It is therefore essential to have in mind a clear picture of the nature of the injuries of the spinal cord which accompany lesions of the vertebral column. In the consideration of these we find the basis for the attitude of extreme conservatism which governs treatment and the reasons for our intention to restrict to the minimum any operative procedures performed in the early stages of spinal injury.

The symptoms of a lesion of the spinal cord which appear simultaneously with the receipt of any injury may be due either to spinal concussion alone, or to a partial or complete contusion or laceration of the cord. In all cases, however, these latter and more serious injuries are at first associated with concussion, which obscures the signs of the underlying injury. The initial clinical picture may, therefore, be identical in all cases, but with the passage of time an uncomplicated concussion will resolve completely, whereas if the concussion masks an underlying contusion of the cord the signs of this injury will only become manifest as the concussion passes off.

Spinal concussion therefore demands initial and careful consideration. It arises as the result of a momentary acute bending of, or a sudden jar transmitted to, the cord. It is the expression of a sudden deformation of nerve fibres and stretching of fibre tracts, which is alone sufficient to lead to a temporary arrest of their function, without postulating a momentary ischæmia as is suggested in Trotter's modification of Kocher's original hypothesis as the basis for concussion of the brain, indeed as Greenfield* remarks any such theory that the lesions of spinal contusion or concussion " are caused by a momentary ischæmia due to the compression of blood vessels will not stand experimental testing." In addition to the violence associated with fractures of the vertebral column, spinal concussion may also result from jarring transmitted to the cord from violent falls on the head or back, or from the passage of a high velocity bullet close to, but not actually

*J. G. Greenfield, Proc. Roy. Soc. Med., Vol. xxxii, p. 43, 1938. 
involving, the spinal column; it occurs more especially where the bullet strikes a spine or transverse process, or becomes embedded in a vertebral body, without however, causing any displacement of bone which could inflict damage on the cord. It will be recalled that a similar loss of function is seen in the concussion of peripheral nerves when a bullet passes close to, but does not actually damage, the structure of the nerve, or strikes a bone in the vicinity. In the cord it is expressed as an immediate loss of all function, motor, sensory and reflex, below the affected site.

The patient is profoundly shocked, with a low blood pressure, the limbs are paralysed, toneless, with absent reflexes; anæsthesia is complete and sphincter control is lost, retention of urine being an almost invariable accompaniment. Recovery from this state may take place within twenty-four hours or be delayed for a week, or longer; yet such a picture may exist in the absence of any permanent damage to the cord, even where an X-ray shows a metallic fragment which has entered at some distant point lying at the end of its course in close proximity to the spine. Yet in certain cases where operation has revealed no injury to the cord and spinal concussion has been assumed, permanent degenerative changes have ensued with the persistence of the signs of a partial or complete lesion of the cord. In these the kinetic energy dissipated by the missile in the adjacent wound has inflicted changes amounting to contusion on the cord without involving it directly. A large number of such cases have now been reported in which it can be proved conclusively that the bullet or displaced bone has not opened the dura or touched the spinal cord; and in these Claude and l'Hermitte, Rev. de Med., I9I6, p. 535, and Gordon Holmes, B.M.J., Nov. 27, I9I5, p. 769, have demonstrated widespread degenerative changes. Naturally enough, no improvement whatsoever followed laminectomy in these cases.

Spinal contusion. We are apt to visualise the pathological change occurring in spinal contusion as being in the nature of a localised hæmorrhage or bruising, a hæmatomyelia strictly localised to the site of injury. Except in the case of penetrating wounds. a hæmatomyelia is in fact extremely rare and the changes seen in spinal contusion are for the most part microscopic and widespread throughout several segments of the cord. Undoubtedly they may result in part from a momentary acute compression, as, for instance, in the displacement accompanying fracture-dislocations; but they also represent the sequelæ of the sudden acute stretching and rupture of fibre tracts, a factor which explains the cases referred to in which permanent atrophic changes may follow the passage of a missile close to, but not directly involving, the cord. These degenerative changes may be observed in both the grey and white matter: in the former capillary hæmorrhages are seen, and in some of the nerve cells the nucleus assumes an eccentric position and stains darkly. In the white matter finely fissured fractures suggest the tearing away of the pia mater and widespread degenerative changes affect the fibre tracts. These extend both up and down the cord from the site of greatest injury and are due to an odema which may be detected clinically in some cases. Thus, as the œdema increases after the initial injury the upper level of the cord lesion may be seen to rise several segments, and this rise in the case of lower cervical or upper dorsal paralysis may prove fatal from the onset of delayed respiratory paralysis, as the phrenic segments gradually become involved. The commonest cause of such an injury is, of course, a fracture-dislocation of the spine, which it will be recalled results from a violent flexion, snapping the vertebral column at the junction of a fixed and a mobile portion, that is, at either the cervico-dorsal or dorsi-lumbar junction. The displacement forwards of the upper vertebra sliding downwards and 
forwards on the lower segment produces a sudden acute compression of several segments of the cord, with an instantaneous partial or complete lesion which is irreparable.

The extent of the damage inflicted on the cord is not, however, governed solely by the simple factor of the degree of displacement of the fracture, but is profoundly modified by the relationship of this fracture to the arteries which supply the cord. Contrary to our previously accepted belief, the spinal arteries do not receive regular reinforcement from lateral branches accompanying each set of nerve roots. There are probably a variable number of larger nutrient vessels each of which supplies many segments. Quite a small fracture which damages such a vessel will have extremely serious neurological sequelæ, whereas a greater displacement which does not damage a nutrient vessel may have less serious results.

In the cervical spine, also, the large size of the neural canal in relation to the nervous tissue allows of considerable displacement occurring without any cord compression whatsoever. For this reason spinal contusion is less common as a complication of cervical dislocation than is the case with fracture-dislocations at a lower level, but when it occurs it is likely to prove fatal from respiratory paralysis.

Spinal laceration. When penetrating wounds involve the spine it will sometimes happen that the damage as seen at operation does not seem severe. There is perhaps a minute speck of bruising visible where a piece of bone has been driven in, but otherwise the appearances are fairly normal; it must however be realised that here again a diffuse contusion of the cord coexists and is likely to be followed by widespread degenerative changes of which the extent of the local bruise is no exact index. More usually there is, unfortunately, less room for doubt for considerable lengths of the cord are completely destroyed. The divided ends are firm and rounded and covered with clots, and between these a custard-like mixture of diffluent cord and broken bone fragments is visible.

Clinically, the presence of an open wound from which cerebro-spinal fluid is discharging is, therefore, of very serious prognosis, but in the case of closed injuries the association of spinal concussion either occurring alone, or masking a more serious underlying injury makes an accurate diagnosis impossible; all that can be said is that the persistence of any sensation or movement is a good sign indicating that the lesion cannot be complete. Here then is the basis of our conservatism. We do not know in certain cases how serious is the injury to the cord, and since we cannot heal that injury, our object in the initial treatment should be to avoid any step which might increase it. The disturbance of an operation to remove a metallic fragment lying near the spine, or to elevate a portion of depressed bone in a closed injury, besides being actively dangerous in a severely shocked patient, will secure no relief if the cord is divided, may increase the cord damage in a partial lesion, and is not required if the cord injury happens to be one of concussion alone. Laminectomy should therefore only be performed in these cases when the patient has recovered from his shock and when the nature of the spinal cord lesion has become manifest; and only then if there is clear evidence of a partial lesion of the cord associated with a spinal block as shown by lumbar puncture and a delayed Queckenstedt's test.

\section{Treatment.}

The immediate treatment of spinal injuries will therefore consist of efforts to limit the amount of cord injury resulting from the displacement of closed fractures, 
and in the case of occasional compound injuries, to obtain and close a surgically clean wound. Elaborate operations for laminectomy have no place in initial treatment. It is perhaps fortunate that the treatment should consist of simple measures for the speed of open warfare imposes obvious restrictions on surgical endeavour. Suggestions which envisage elaborate procedures may be extremely practical in the treatment of civilian casualties but in open warfare with the ebb and flow of battle the position of Casualty Clearing Stations must vary at times between equally undesirable extremes of remoteness and proximity. Ideals may therefore be impossible to maintain; speed demanding relatively simple methods of treatment.

In the first place, the bearers should be instructed in every paralysed case of spinal injury to do nothing that might increase the injury of the cord; since the majority of fractures result from flexion, the patient should be carried with the spine in an extended position. Flexion is to be avoided absolutely in all spinal fractures since it will sometimes cause the upper segment of the spine to slip forward on the lower, thus acutely compressing the cord and causing a complete transverse lesion in a patient who previously had no more than spinal concussion or'a partial contusion. To avoid this accident cases should never be lifted by the feet and shoulders but should be carefully turned face downwards on to a blanket opened and placed on a stretcher. If an open wound is present a firstaid dressing is applied and the patient should then be covered warmly on account of shock, a folded coat or blanket placed under the chin to increase extension, and the patient brought to the aid post in this position. The blanket under the patient provides a most useful sling in which he may be kept in a position of extension as he is lifted either on to an operating table or a bed.

In both closed and open injuries shock should be treated first by the usual measures of warmth and morphia, supplemented by transfusion if external bleeding has been severe. Where an open wound exists we have to strike a balance between the risk of septic infection which increases with each hour that elapses prior to excision and surgical cleansing of the wound, and the risk of operating on a gravely shocked patient. In general it will be found that time spent in the treatment of shock will be well repaid, and during this time as a first-aid procedure any obvious bleeding vessels should be ligated and a pack of euflavine $I: 2,000$, or aqueous azochloromide $I: 3,000$, should be placed in the wound and a firm dressing applied pending operation. These antiseptics are said to have no harmful effect on nervous tissue. When the discharge of cerebro-spinal fluid from the wound indicates that the meninges are open, two half-gramme tablets of M. \& B. 693 should be given to the conscious patient, or in the unconscious 20 c.cs of $2 \frac{1}{2}$ per cent. streptocide should be injected intramuscularly with a view to decreasing the risk of meningitis, in addition to the routine injections of anti-tetanic and anti-gas gangrene serum. When fit enough, the patient should be operated upon or transported to a suitable centre. Local anæsthesia is in many cases all that is required since the patient is already anæsthetic. The introduction of $\frac{1}{2}$ per cent. novocaine with a small quantity of I:I,000 adrenalin does, moreover, diminish bleeding from the paraspinal muscles which may otherwise be extremely tiresome. The wound must then be completely excised to its depth, hæmorrhage from the muscles being arrested by warm packs, provided that hot saline is not allowed to come in contact with the cord. If the dura is open, dirt and clot may be removed from the surface of the cord by gentle washing with saline and suction to prevent the dirt being washed in. Loose bone fragments should be gently removed. Bleeding from the epidural veins may be arrested with muscle implants and the wound closed by careful suture in three tiers. It is not necessary to attempt to suture the dura, provided surface 
suturing is efficiently carried out. It is, of course, unnecessary to remark that even in the case of the cauda equina which it is sometimes suggested should be sutured, suture of divided nervous tissue is futile and should not be considered. Even in the cauda the absence of a neurilemma precludes regeneration.

The after-treatment of these cases follows that adopted in closed injuries. It must be admitted, however, that operations of this type will seldom be required. Few cases of compound injury with exposure of the cord survive long enough to come to active treatment; where they do so the results of operation will be disappointing, for the damage inflicted on the cord at the time of injury is fixed and permanent and is not influenced by the subsequent removal of bone fragments and clots. Further, the risk of meningeal infection is high. The occasional success will be found in the case of the wound which is explored and is shown not to involve the spinal cord at all; examples of spinal concussion and in those cases where the lesion is only partial and in which the onset of sepsis can be avoided. Disappointment due to the occurrence of delayed atrophy in cases where concussion had been assumed will however have to be faced.

\section{Immobilisation.}

In closed injuries and in all cases where the spine is fractured, some form of reduction and immobilisation will be required. Plaster is the method of choice in many cases and should certainly be employed in the treatment of civilian injuries or where sufficient time is available on active service. A method for temporary immobilisation which may be used during transport is a Liston's long splint applied to the back, but this, of course, does not provide the hyperextension which is required.

Immobilisation should not be carried out until the shock has been treated. An $\mathrm{X}$-ray picture is then a useful accessory, but no undue movement of the patient should be undertaken merely to obtain a picture. The same remark applies to neurological examination. We can find out all we want to know by the simplest tests. Is the patient conscious of pin-pricks? Test the ankle jerks and plantar responses. The persistence of any normal function must indicate that the lesion is only partial; complete loss of function may still be due to spinal concussion.

Immobilisation in fracture-dislocations of the spinal column should be performed in the extended position and this is where the value of a blanket placed under the patient becomes apparent. He may be lifted in this sling, the sag of which produces extension. The ends of the blanket should then be fixed to two tables. Approximation of these tables one to the other increases the extension resulting from the sag. When a suitable position has been obtained, a plaster case with reinforcing bars of plaster may be applied enclosing the patient, together with portions of the blanket, and when this is dry he may then be turned over without risk of the fracture slipping. It is obvious, however, that this somewhat elaborate method can hardly be employed during the intense rush accompanying major engagements, until the patient has been transported some distance towards the base.

The treatment of cervical fractures and dislocations demands special consideration. It may well be that pain and deformity resulting from such injury is not accompanied at first by neurological sequelæ, but this does not mean that signs of cord compression may not appear later and, in some cases, dramatically. If a fracture-dislocation of the cervical spine is detected, immobilisation must be secured 
by plaster, or splintage, before the patient is sent down the line. I have recently seen a number of casualties treated as walking wounded who developed signs of cord compression three days or more after the receipt 'of their injury. Pure dislocations of the cervical spine may be reduced by manipulation, using Walton's manœuvre in which a flannel sling is passed under the patient's jaw and occiput and tied behind the operator's back, so that by leaning backwards an extending force is produced leaving the two hands free for the lateral rotation necessary to produce reduction. Flexion must be carefully avoided during this manœuvre, which will require deep anæsthesia for its success.

If the spine is fractured as well as dislocated, manipulation may be actively dangerous. Immobilisation should be secured for transit and reduction should only be attempted either by the method of a head halter or skull traction after the patient has been brought to a base hospital where continuous treatment can be given. The length of time for which immobilisation will be required varies with the site of the fracture; six weeks is usually sufficient in the cervical spine, but fractures of the vertebral column may require immobilisation for a period of from three to five months.

\section{After-Treatment.}

The phases of spinal injury have been described as falling under three periods, namely, the stages of spinal shock, return of reflex activity, and of septic complications. When immobilisation has been secured, we await the subsidence of the spinal shock so that we may judge from the character of the reflex activity that returns what is the exact injury which the cord has suffered, and meanwhile use our best endeavours to prevent the septic complications of bed sores and urinary infection.

Concussion passes off in twenty-four hours to one week. Persistence of sensory loss or paralysis beyond this period indicates a partial or complete lesion of the cord.

Reappearance of sensation and motor power, occasionally producing a BrownSéquard syndrome, of course, indicates a partial lesion. The character of the paraplegia in partial lesions is of the extensor type, owing to the action of the extrapyramidal pathways continuing to restrain the lower spinal flexor reflexes.

In complete lesions of the cord, not only is there no recovery of motor or sensory function or sphincter control, but also when the spinal concussion resolves we find that the patient shows a paraplegia in flexion owing to the release of the spinal reflexes from any higher control.

For this reason, also, the reflexes of spinal automatism become manifest, of which the commonest example is the mass reflex; flexion of the ankle, knee and hip, sometimes accompanied by evacuation of the bladder, produced by pinching the skin of the dorsum of foot or leg. Spontaneous flexor spasms also appear and may be deceptively encouraging. It must be remembered, however, that these movements result from uninhibited spinal reflexes frequently caused by the touch and pressure of bedclothes and far from indicating any recovery of function, are a sign of gross damage to the cord. These spasms may prove extremely tiresome and frequently necessitate the removal of the plaster shell, as they lead to the production of sores either over the front of the thighs or, in the case of adductor spasm, on the inner surface of the knees. 
The precise character of the signs observed will vary according to the level of the lesion. In cervico-dorsal dislocations the signs are all upper motor neuron in type. In dorsi-lumbar fractures lesions of the conus medallaris or cauda equina produce signs which may be purely lower motor neuron or a mixture of upper and lower motor neuron, according to the situation of the fracture.

\section{Treatment of the Bladder.}

Retention of urine is an invariable accompaniment of the phase of spinal shock. The effect of partial or complete injuries on the function of the bladder centres varies with the level of the lesion. As the spinal shock passes off in cervico-dorsal injuries, the lesion being above the bladder centres, an automatic periodic reflex micturition is established; whereas in lesions of the cauda equina paralysis of the detrusor muscle results in a permanent retention, with overflow. Since, however, these latter patients regain the power of contracting their abdominal muscles they can strain and in this way expel some of the urine retained in the bladder, which, however, is never completely emptied. In this way they can keep fairly dry during the day but at night dribbling incontinence will again manifest itself. It will be seen that the initial phase is always one of retention, carrying with it the risk of infection from catheterisation and ascending infection from high intravesical pressure; nor is the risk of infection removed when either automatic action or the power of voluntary expression is regained; indeed in a discussion on this subject Kenneth Watkins (Proc. Roy. Soc. Med., vol. xxx, I937, p. I290) gives it as his opinion that ascending infection is more liable to occur with true automatic action than in the less active bladder resulting from lesions of the conus or cauda equina. The care of the bladder is therefore an extremely important matter. In IgI9, Thomson Walker (I9I9 B.M.J. Part I, p. 325) estimated the total death rate due to urinary sepsis in spinal cases as 80 per cent., a figure which is due almost entirely to the introduction of infection by repeated catheterisation. To remove this risk three methods are available-manual expression, an indwelling catheter with repeated irrigation, or a prophylactic suprapubic cystostomy.

Manual expression consists of gentle but firm massage of the bladder through the abdominal wall. When the patient is lying on his back this is easy to perform, owing to the paralysis of the parietal musculature in most lesions ; it should, however, be repeated every four to six hours in order to avoid over-distension, which, strangely enough, causes pain in certain cases, presumably via sympathetic pathways which enter the cord above the site of the lesion. In some cases contraction of the bladder sphincter may defeat our efforts at expression and then requires additional measures such as morphia or warmth to the perineum together with doryl.

This method although spoken of very highly by some writers is not without its difficulties and occasionally its risk-that of rupture of the bladder. Vellacott and Webb Johnson quote this complication in one of their sixteen cases (I9I9 Lancet, vol. I, p. 733). It certainly does not interfere in any way with the development of reflex micturition but when infection has become established in the bladder it is of course harmful in causing regurgitation of septic urine along the lumen of the ureter to the renal pelvis (Sir John Thomson Walker, Proc. Roy. Soc. Med. vol. xxx, I937, p. I237). The method is, however, of value and should be tried at first, and repeated at regular intervals if successful.

Where the patient has to be transported over long distances prior to immobilisation, as sometimes occurs under service conditions, an indwelling catheter should 
be passed to provide continuous drainage into a bottle, with daily bladder irrigation ; special care being required to prevent the catheter slipping out during transit, as, if this happens, intermittent catheterisation is likely to be used instead, with all the risks of sepsis being introduced. An indwelling catheter will also be required if manual expression fails after the patient has been successfully immobilised. Cystitis is, of course, likely to occur, but provided that a rise of intravesical pressure is prevented by continuous drainage, ascending infection of the kidneys is unlikely. For this reason the catheter must never be clipped or plugged. When the patient has arrived at a hospital where continuous treatment can be given, the catheter should be attached to a Y-tube, one limb of which passes to the collecting bottle and the other to a reservoir of irrigating fluid. Frequent irrigation can then be carried out simply by placing a clip on the tube leading to the receptacle and opening the clip which shuts off the reservoir, as in the Duke's apparatus.

Suprapubic cystostomy has been suggested as a method for immediate use as a prophylactic against ascending infection. It should certainly be performed when it is obvious from the other signs that the patient has a spinal lesion which is sufficiently grave to render a long period of retention probable and where manual expression cannot be maintained. But this diagnosis cannot be made for the first week or so in many cases owing to the spinal concussion. For this reason, an indwelling catheter provides a useful intermediate step during this period. Suprapubic cystostomy should be performed also when cystitis or severe urethritis is present, since the latter complication may lead to extensive sloughing and fistula formation around an indwelling catheter. It must be remarked, however, that when the skin of the abdominal wall is anæsthetic, sloughing is liable to occur around a cystostomy opening. It does not appear right, therefore, to perform cystostomy as an immediate step in Casualty Clearing Stations in every case, but only after other methods such as manual expression have been tried and due consideration given to the pros and cons of every case. It will, however, be required and prove valuable in many cases. It should be performed by the Clifford Morson technique, using a trocar and cannula along the lumen of which a self-retaining catheter stretched over a stylet is introduced into the distended bladder immediately above the symphysis pubis.

When reflex activity returns the suprapubic fistula, if formed by this method, heals readily when the tube is removed. It is important to realise how long it may be before such activity is likely to commence. Sir John Thomson Walker (ibid) in reviewing his extensive experience gives the duration of the stage of retention as varying between twenty-four hours and eighteen months, with an average duration in thirty consecutive cases of fifty-five days. It is for this reason that, if manual expression fails, suprapubic cystostomy is.likely to be required in order to prevent infection.

Restoration of function. It has been suggested that in certain cases of partial lesions of the sacral cord or cauda equina where retention persists despite an otherwise satisfactory recovery of motor and sensory function, it is possible to establish normal micturition by performing a pre-sacral neurectomy. The theory underlying this procedure is that the nervi erigentes supplying the detrusor muscle are weakened, but not completely out of action: they are, however, overbalanced by the action of the opposing inhibitory sympathetic control running in the pre-sacral nerve. The removal of these fibres is said to restore the balance of power in favour of the weakened nervi erigentes, thus allowing the detrusor muscle to overcome the resistance of the sphincter and empty the bladder. The selection of suitable cases requires careful investigation by cystometric tests. I have carried out this operation 
with improvement, and, I believe, with success, but in assessing these results we have to bear in mind the long period within which normal recovery may occur independently of operative procedures. The value of this operation is one of the minor surgical problems on which we can expect the experience of this war to shed further light.

In partial spinal lesions it must be borne in mind that the recovery of muscular power is an extremely slow and gradual process extending in certain cases over several years. During this period everything possible must be done to encourage movement; massage is of use to reduce rigidity, and the limbs should be put daily through the full range of normal movement to prevent contracture. Splintage and cradling to prevent such complications as foot drop and the over-stretching of antagonists should, of course, be employed. Electrical treatment is valueless in upper motor neuron lesions and should not be used except in the case of partial lesions of the cauda equina. For the restoration of muscular power a single voluntary contraction is worth a deal of massage and passive movement, but the paralysed limb is a heavy object for weakened muscle groups to move. Exercises carried out in a warm water tank in which the patient is supported on a canvas sling so that he lies in the surface of the water-a method used in the treatment of poliomyelitis in children is of the utmost value. The buoyancy imparted to the limb enables movement to be carried out which would be quite impossible with the patient lying in bed. It is to be hoped that such facilities will be available. Finally, when the end result has really been achieved as regards the recovery of the spinal cord, we have the valuable aid of orthopædic surgeons, who, by tenotomy and arthrodesis can do so much to improve still further the mechanical function of the limb. 